\title{
THE CONTROL OF DRILLING VIBRATIONS: A COUPLED PDE-ODE MODELING APPROACH
}

\author{
Belem SALDIVAR $^{a, *}$, SABINE MONDIÉ $^{b}$, JUAN CARlos ÁVILA VILCHIS $^{a}$ \\ ${ }^{a}$ Faculty of Engineering \\ Autonomous University of the State of Mexico, Instituto Literario No. 100 Ote., 50130 Toluca, Mexico \\ e-mail: mbsaldivarma@conacyt.mx, jcavilav@uaemex.mx \\ ${ }^{b}$ Department of Automatic Control \\ CINVESTAV-IPN, Av. IPN No. 2508, Col. San Pedro Zacatenco, C.P. 07360, México D.F., Mexico \\ e-mail: smondiedctrl.cinvestav.mx
}

\begin{abstract}
The main purpose of this contribution is the control of both torsional and axial vibrations occurring along a rotary oilwell drilling system. The model considered consists of a wave equation coupled to an ordinary differential equation (ODE) through a nonlinear function describing the rock-bit interaction. We propose a systematic method to design feedback controllers guaranteeing ultimate boundedness of the system trajectories and leading consequently to the suppression of harmful dynamics. The proposal of a Lyapunov-Krasovskii functional provides stability conditions stated in terms of the solution of a set of linear and bilinear matrix inequalities (LMIs, BMIs). Numerical simulations illustrate the efficiency of the obtained control laws.
\end{abstract}

Keywords: drilling vibrations, LMI approach, ultimate boundedness, coupled wave-ODE system.

\section{Introduction}

Vibrations in mechanical systems are oscillations occurring without being intentionally provoked that often have adverse effects on the system performance. In oilwell drillstring systems, vibrations occurring along the rod constitute a major source of economic losses since they cause premature wear and failures of the system components and prolong the drilling time.

During the drilling process, the interaction between the cutting device (called the bit) and the rock formation gives rise to the occurrence of three types of vibrations:

- Torsional vibration. This self-excited rotational motion, also known as stick-slip, is induced by the nonlinear relationship between the torque and the angular velocity at the bit (Jansen and van den Steen, 1995). The torsional flexibility of the drilling assembly exacerbates nonuniform oscillatory behavior causing rotational speeds of more than twice the nominal rotary table speed or a total standstill of the bit (Skaugen, 1987).
- Axial vibration. It is generally induced by torsional vibration and consists of irregular movements of the drilling components along a longitudinal axis causing bit-bounce and rough drilling behavior.

- Lateral vibration. Deep in the hole, the rotating drillstring interacts with the borehole wall generating shocks from lateral vibrations (whirling). Lateral vibration damages the borehole wall and affects the overall drilling direction (Jansen, 1993).

Extensive research effort has been into the modeling and control of drilling vibrations put over the past century. Before the 1960s, investigations were focused on material strength of the system components, but the trends have changed to emphasize dynamic behavior (Jansen, 1993). In 1960, Bailey and Finnie of Shell Development Company developed the first analytical and experimental study on torsional and axial drilling vibrations (Bailey and Finnie, 1960). Since then, numerous approaches for modeling and control have been proposed. The most popular control techniques are described below. 
Steering torque feedback system (Halsey et al., 1988). The underlying idea is to adjust the angular velocity according to torque variations; hence, propagating waves are dampened at the top extremity instead of being reflected back to the drillstring. The major shortcoming of this strategy is that it requires an accurate measurement of the torque, which, in practice, can be difficult to obtain.

Soft torque rotary system (STRS) (Javanmardi and Gaspard, 1992). This method is an improved version of the feedback torque technique. It avoids the task of measuring the drillstring torque by computing it through the motor current. A proper tuning of the controller allows reducing drillstring vibrations.

Proportional-integral-derivative (PID) control (Pavone and Desplans, 1994). This is a simple strategy to avoid the stick-slip phenomenon. PID controller gains are obtained through an appropriate stability analysis. A drawback of this technique is that the drillstring vibrations are not sufficiently damped to guarantee an optimal drilling performance.

$\mathcal{H}_{\infty}$-controller (Serrarens et al., 1998). Results obtained with experimental prototypes have shown that torsional drilling oscillations are reduced by means of an $\mathcal{H}_{\infty}$ control law which is linear, time-invariant and has robust qualities. However, in order to get a proper control performance, a very accurate model is required. Another disadvantage of this method is that saturation constraints are not well-handled.

Active vibration damper (Jansen and van den Steen, 1995). The basic idea of this method consists in increasing the viscous damping at the bottom end to avoid drillstring vibrations. The damping coefficient is modified via a magnetorheological fluid, which allows manipulating the viscous properties of the drilling mud. This strategy allows attenuating the stick-slip vibrations; however, an optimal drilling operation requires additional control actions.

Sliding mode control (Navarro-López and Licéaga-Castro, 2009). This control strategy, introduced by Navarro-López and Cortés (2007b) and discussed and modified by Navarro-López and Licéaga-Castro (2009), is based on the bifurcation analysis of a lumped parameter model describing the torsional drilling dynamics developed by Navarro-López and Cortés (2007a). The stick-slip phenomenon can be mathematically seen as a sliding motion which occurs when the bit velocity is zero. The existence of this sliding motion depends on the weight on the bit and the torque applied by the surface motor. Such a regime is the main cause of bit sticking problems. The sliding mode control consists in introducing another discontinuity surface and forcing the system to evolve along it. On the new surface, the bit speed will follow the top-rotary-system speed after reasonable time, avoiding the bit sticking phenomena. This strategy does not represent an automatic controller, but it should be understood as an off-line safe parameters selection method which helps the driller operators avoiding bit sticking problems.

D-OSKIL (Canudas-de Wit et al., 2008). D-OSKIL is a short word for the drilling oscillation killer. This method uses the weight on the bit as an additional control variable. The control proposal is based on the fact that a sufficiently large weight on the bit is required to guarantee a satisfactory rate of penetration, and if it reaches higher values, drilling vibrations may arise. An optimal trade-off between the weight on the bit and the rate of penetration has to be found. Experimental implementation of such a mechanism, in a laboratory testbed, is reported by $\mathrm{Lu}$ et al. (2009). One disadvantage of this method is that its implementation may require repetitive addition and removal of drill collar sections to properly adjust the control law, which may come out infeasible and could induce axial vibrations.

Flatness-based control (Saldivar et al., 2014). The differential flatness property of the drilling system is exploited to design a pair of stabilizing controllers tackling the trajectory tracking problem. This approach gives rise to an exponential convergence of the error toward zero, allowing the suppression of undesirable drillstring vibrations. By means of the flatness-based controllers proposed by Saldivar et al. (2014), the stick-slip and the bit-bounce are substantially reduced in a relatively short period of time; however, the price paid for control effectiveness is its structural complexity.

Despite the development of numerous methods for eliminating drilling vibrations, nowadays such phenomena still greatly affect perforation processes. This is mainly due to the lack of proper understanding of the system's dynamics; in fact, most of the proposed techniques are based on simplified lumped parameter models that disregard the distributed nature of the system and only consider torsional drilling behavior.

The aim of this contribution is the improvement of the drilling performance through the design of appropriate stabilizing control laws for the elimination of the most frequently observed vibration modes: torsional and axial oscillations. The control design proposal is based on a reliable drilling system model which allows considering its distributed nature. An infinite-dimensional model, given by a partial differential equation (PDE), is used to describe the torsional waves traveling along the rod. Through the torque on the bit function which approximates the frictional interface between the cutting device and the drilling surface, the PDE model is coupled to an ordinary differential equation (ODE) representing the drillstring axial excitations. 
Several control methods to stabilize systems described by coupled PDEs have been proposed over the last decade (see, e.g., Wu et al., 2010; Yang and Wang, 2014; Zhang and Zuazua, 2004; Zhou and Tang, 2012). The most popular strategies include Lyapunov analysis (Suh et al., 2006), backstepping (Ma et al., 2013) and forwarding techniques. Despite the extensive literature on the analysis and control of distributed parameter systems, little attention has been paid to an effective and simple technique allowing stabilization of dynamic systems: the LMI method.

In this contribution, we propose a novel systematic method for designing control laws aimed at stabilizing the coupled PDE-ODE system considered. The strategy is based on Lyapunov stability theory; a pair of conditions on a Lyapunov-Krasovskii functional proposal and its derivative allows determining an ultimate bound for the system trajectories. These stabilizing conditions are stated in terms of matrix inequalities, which come out feasible through a suitable choice of the controllers structure.

The performance of the proposed method is highlighted through simulations of the drilling system considering numerical parameters that reflect typical operating conditions in real oilwell drilling platforms. It will be shown that the obtained control laws are effective in eliminating coupled drilling vibrations. Furthermore, a comparative analysis of the system response under two different control solutions, the soft-torque controller and the flatness-based approach, is developed. Simulation results show an improved performance of the control method presented in this contribution compared with the specific controllers presented by Tucker and Wang (1999) as well as Saldivar et al. (2014).

The paper is organized as follows. Section 2 introduces the proposed modeling strategy; a nonlinear coupled wave-ODE system describes the torsional-axial behavior of the drilling rod. Section 3 provides sufficient conditions on a Lyapunov-Krasovskii functional guaranteeing ultimate boundedness of the system solutions. Section IV presents a strategic methodology allowing the design and synthesis of stabilizing feedback controllers; the obtained stabilization conditions are stated in terms of the solution of an LMI-BMI feasibility problem. In Section 5, two popular control strategies to reduce drilling vibrations are reviewed: the soft-torque rotary system and the flatness-based control approach. A comparative analysis based on simulations of the drilling system allows highlighting the effectiveness of the BMI-based control approach in suppressing drilling vibrations. Concluding remarks are given in Section 6.

Notation. Throughout the paper the superscript $T$ stands for matrix transposition, $\mathbb{R}^{n \times m}$ is the set of all $n \times m$ real matrices, the notation $P>0(P<$ 0 ), for $P \in \mathbb{R}^{n \times m}$ means that $P$ is symmetric and positive (negative) definite, whereas $\lambda_{\max }(P)$ denotes its maximum eigenvalue. The symmetric elements of the symmetric matrix are denoted by $*$. The notation used for partial derivatives is as follows:

$$
\begin{aligned}
z_{\zeta}(\zeta, t) & =\frac{\partial z}{\partial \zeta}(\zeta, t), \\
z_{t}(\zeta, t) & =\frac{\partial z}{\partial t}(\zeta, t), \\
z_{\zeta \zeta}(\zeta, t) & =\frac{\partial^{2} z}{\partial \zeta^{2}}(\zeta, t), \\
z_{t t}(\zeta, t) & =\frac{\partial^{2} z}{\partial t^{2}}(\zeta, t), \\
z_{\zeta t}(\xi, t) & =\frac{\partial^{2} z}{\partial \zeta \partial t}(\zeta, t) .
\end{aligned}
$$

\section{Drilling system modeling}

A sketch of a simplified drillstring system is shown in Fig. 1.

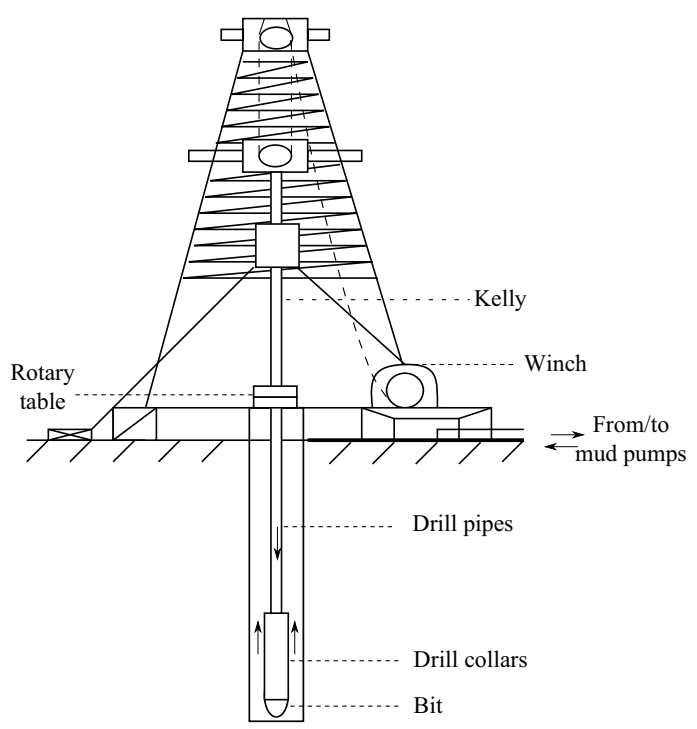

Fig. 1. Basic scheme of a vertical drilling system.

The main process during well drilling for oil is the creation of borehole by a rock-cutting tool called a bit. The drillstring consists of the bottom hole assembly (BHA) and drill pipes screwed end to end to each other to form a long pipe. The BHA comprises the bit, stabilizers to prevent the drillstring from unbalancing, and a series of heavy pipe sections which are known as drill collars. We are particularly interested in analyzing two main phenomena damaging the drilling system components and delaying the overall perforation process: the torsional and axial drilling vibrations. Torsional vibrations (stick-slip phenomenon) are essentially the cause of premature 
breakage of drill pipes. Axial vibrations (bit-bouncing phenomenon) may severely damage the cutting device. Self-excited axial and torsional oscillations are intimately coupled and may occur simultaneously. The complex nature of coupled drilling vibrations makes the derivation of a model mathematically challenging and is one of the reasons why the full system has been rarely considered so far.

This section presents a mathematical model that comprises the coupled torsional and axial drilling dynamics.

The propagation of torsional waves along a drillstring of length $L$ can be modeled by the damped wave equation (Challamel, 2000; Fridman et al., 2010; Saldivar and Mondié, 2013; Tucker and Wang, 1999)

$$
\begin{gathered}
G J \frac{\partial^{2} z}{\partial \xi^{2}}(\xi, t)-I \frac{\partial^{2} z}{\partial t^{2}}(\xi, t)-\gamma \frac{\partial z}{\partial t}(\xi, t)=0, \\
\xi \in(0, L), \quad t>0,
\end{gathered}
$$

where the twist angle $z$ depends on length coordinate $\xi$ and time $t$. The parameters $G$ and $J$ are the shear modulus and the polar moment of inertia, respectively. The distributed inertia moment $I$ is such that $I=\rho_{d} J$, where $\rho_{d}$ is the mass density of the drillstring. A distributed viscous damping $\gamma>0$ is assumed along the structure. The mechanical model is derived from the equations describing torsional vibrations of circular shafts studied by Timoshenko and Young (1955) as well as Weaver et al. (1990).

An appropriate choice of boundary conditions allows characterizing the propagating torsional waves along the drillstring. In the work of Saldivar et al. (2013), the following boundary conditions are introduced:

$$
\left\{\begin{array}{l}
G J \frac{\partial z}{\partial \xi}(0, t)=c_{\mathrm{a}}\left[\frac{\partial z}{\partial t}(0, t)-\Omega(t)\right] \\
G J \frac{\partial z}{\partial \xi}(L, t)=-I_{B} \frac{\partial^{2} z}{\partial t^{2}}(L, t)-T\left(\frac{\partial z}{\partial t}(L, t)\right) .
\end{array}\right.
$$

It is assumed that the drillstring is rotated from the surface $(\xi=0)$ by an electrical motor providing an angular velocity $\Omega(t)$. Due to the kinematic chain between the rotor and the drillstring upper extremity, $\Omega(t)$ does not match the rotational speed of the load $\frac{\partial z}{\partial t}(0, t)$; this sliding speed gives rise to the local torsion of the drillstring. The parameter $c_{\mathrm{a}}$ denotes the angular momentum at the top extremity.

The inferior extremity is subject to a torque $T$ which is a function of the bit speed $\frac{\partial z}{\partial t}(L, t)$. A lumped inertia $I_{B}$ is chosen to represent the assembly at the bottom hole.

Torsional dynamics are controlled through the angular velocity $\Omega(t)$ imposed at the surface.

Axial dynamics of the drillstring can be described by an ordinary differential equation (Challamel, 2000).
The modeling strategy is inspired by the fact that any mass subject to a force in a stable equilibrium acts as a harmonic oscillator for small vibrations. More precisely, the damped harmonic oscillator model describing the longitudinal drillstring motion is

$$
m_{0} \ddot{y}+c_{0}[\dot{y}+\Gamma(t)]+k_{0} y=-\mu_{1} T\left(\frac{\partial z}{\partial t}(L, t)\right),
$$

where the variable $y$ is defined as $y=Y-\Gamma_{0} t, m_{0}, c_{0}$, and $k_{0}$ represent the mass, damping and spring constant, respectively, $Y$ denotes the bit axial position. The system is controlled through the rate of penetration $\Gamma(t)$, which is an axial speed imposed at the surface, and $\Gamma_{0}$ is a constant nominal value.

According to Detournay and Defourny (1992), the coefficient $\mu_{1}$ can be obtained with the following relation: $\mu_{1}=2\left(R_{b} \mu_{\text {bit }} c_{\mathrm{bit}}\right)^{-1}$, where $R_{b}$ is the bit radius, $\mu_{\text {bit }}$ is the friction coefficient at the bit-rock contact, and $c_{\text {bit }}$ is the so-called bit coefficient. For a bladed bit, $c_{\mathrm{bit}}$ is equal to the dimensionless length of the cutting edge (and independent of the number of blades). For a flat bit, $c_{b i t}$ is computed as follows: $c_{\text {bit }}=\left(6+4 \rho_{\text {bit }}\right) /\left(6+3 \rho_{\text {bit }}\right)$, where $\rho_{\text {bit }}$ is the radial rate of increase in cutter density. Notice that $c_{\text {bit }}$ varies between 1 and $4 / 3$ and may be considered a constant.

The coupling between subsystems is due to the torque on bit $T$ approximating the physical phenomena at the bottom end:

$$
T\left(\frac{\partial z}{\partial t}(L, t)\right)=c_{\mathrm{b}} \frac{\partial z}{\partial t}(L, t)+T_{\mathrm{nl}}\left(\frac{\partial z}{\partial t}(L, t)\right) .
$$

The model of $T$ includes a linear term $c_{\mathrm{b}} \frac{\partial z}{\partial t}(L, t)$ representing the viscous damping torque which approximates the influence of the mud drilling and a nonlinear term $T_{\mathrm{nl}}\left(\frac{\partial z}{\partial t}(L, t)\right)$ representing the dry friction torque which models the bit-rock contact. It is assumed that the growth of instabilities leading to harmful drilling vibrations arises from the friction torque which can include velocity weakening laws as in the work of Challamel (2000), stiction plus Coulomb friction (Jansen and van den Steen, 1995; Serrarens et al., 1998), and the Stribeck effect, characterized by a decreasing friction-velocity map localized around zero velocity, as in the following model introduced by Navarro-López and Suárez (2004):

$$
\left\{\begin{array}{c}
T_{\mathrm{nl}}=W_{\mathrm{ob}} R_{\mathrm{b}} \mu_{\mathrm{b}}\left(\frac{\partial z}{\partial t}(L, t)\right) \operatorname{sign}\left(\frac{\partial z}{\partial t}(L, t)\right) \\
\mu_{\mathrm{b}}\left(\frac{\partial z}{\partial t}(L, t)\right)=\mu_{\mathrm{cb}}+\left[\mu_{\mathrm{sb}}-\mu_{\mathrm{cb}}\right] \mathrm{e}^{-\gamma_{\mathrm{b}}\left|\frac{\partial z}{\partial t}(L, t)\right|}
\end{array}\right.
$$

where $W_{\mathrm{ob}}>0$ is the weight on the bit, $\mu_{\mathrm{sb}}, \mu_{\mathrm{cb}} \in(0,1)$ are the static and Coulomb friction coefficients and $0<$ $\gamma_{\mathrm{b}}<1$ is a constant defining the velocity decrease rate. 
Consider the torsional model (1)-(2) coupled to the axial one (3) through the torque on the bit function (4)-(5). In order to take into account external disturbances and unmodeled dynamics at the lower extremity of the drillstring, we introduce an additive variable $\delta(t)$ into the second boundary condition. For the sake of simplicity, we introduce the normalized rod length $\zeta=\xi / L$. The coupled wave-ODE model reads

$$
\begin{aligned}
z_{t t}(\zeta, t)= & a z_{\zeta \zeta}(\zeta, t)-d z_{t}(\zeta, t), \quad \zeta \in(0,1) \\
z_{\zeta}(0, t)= & g\left[z_{t}(0, t)-u_{1}(t)\right] \\
z_{\zeta}(1, t)= & -h z_{t t}(1, t)-k z_{t}(1, t) \\
& -q T_{\mathrm{nl}}\left(z_{t}(1, t)\right)+\delta(t) \\
\dot{x}(t)= & A x(t)+B u_{2}(t) \\
& +E_{1} z_{t}(1, t)+E_{2} T_{\mathrm{nl}}\left(z_{t}(1, t)\right) .
\end{aligned}
$$

The controllers $u_{1}(t)$ and $u_{2}(t)$ correspond to the angular velocity provided by the rotary table $\Omega(t)$ and to the rate of penetration imposed at the top end $\Gamma(t)$, respectively. The variable $\delta(t)$ is assumed to satisfy

$$
\delta(t)^{2} \leq \varepsilon
$$

with $\varepsilon>0$ small enough. The vector $x(t)$ is defined as $x(t)=[y(t) \dot{y}(t)]^{T}$, the model parameters are given by

$$
\begin{aligned}
& a=\frac{G J}{I L^{2}}, \quad d=\frac{\gamma}{I}, \quad g=\frac{c_{a} L}{G J}, \\
& h=\frac{I_{B} L}{G J}, \quad k=\frac{c_{b} L}{G J}, \quad q=\frac{L}{G J},
\end{aligned}
$$

and the constant matrices are defined as

$$
\begin{gathered}
A=\left[\begin{array}{cc}
0 & 1 \\
-\frac{k_{0}}{m_{0}} & -\frac{c_{0}}{m_{0}}
\end{array}\right], \quad B=\left[\begin{array}{c}
0 \\
-\frac{c_{0}}{m_{0}}
\end{array}\right], \\
E_{1}=\left[\begin{array}{c}
0 \\
-\frac{\mu_{1} c_{\mathrm{b}}}{m_{0}}
\end{array}\right], \quad E_{2}=\left[\begin{array}{c}
0 \\
-\frac{\mu_{1}}{m_{0}}
\end{array}\right] .
\end{gathered}
$$

\section{Ultimate boundedness result}

It is well known that most physical real-world systems exhibit nonlinear characteristics and uncertainties, making the achievement of exponential stability impossible; however, the system response oscillates close enough to the equilibrium to be considered acceptable. In many stabilization problems, the aim is to bring states close to certain sets, rather than to a particular state. In this situation, classical stability notions (for instance, exponential or asymptotic stability) are not appropriate. More suitable performance specifications, from an engineering point of view, are given by the definition of practical stability, also referred to as ultimate boundedness with a fixed bound (Khalil, 2002), introduced by La Salle and Lefschetz (1961) and retaken by Grujić (1973) as well as Lakshmikantham et al. (1990). These definitions not only provide information on the stability of the system, but also characterize its transient behavior with estimates of the bounds on the system trajectories (Anabtawiii, 2011).

It is worth mentioning that the concept of practical stability is also referred to as dissipativeness in the sense of Levinson. This qualitative property is important in the theory of differential equations, especially in the study of self-sustained and forced nonlinear oscillations (Rasvan, 2006).

The dissipativeness definition is stated by Levinson (1944) as follows.

Definition 1. (Levinson, 1944) The system

$$
\dot{x}(t)=f(t, x)
$$

is called dissipative if there exists some $R>0$ such that

$$
\varlimsup_{t \rightarrow \infty}\left|x\left(t ; t_{0}, x_{0}\right)\right|<R
$$

for any solution $x\left(t ; t_{0}, x_{0}\right)$ of 8 .

This qualitative property, also called ultimate boundedness, means that all systems trajectories will eventually enter into a ball of radius $R$ and will remain there. The following result states conditions for uniform Levinson dissipativeness.

Theorem 1. (Yoshizawa, 1960) Assume that there exist $R>0$ and a function $V:\left\{x \in \mathbb{R}^{n}:|x| \geq R\right\} \times$ $\left[t_{0}, \infty\right) \rightarrow \mathbb{R}^{+}$which satisfies

$$
\alpha_{0}(|x|) \leq V(x, t) \leq \alpha_{1}(|x|), \quad \dot{V}(x, t) \leq-\alpha_{2}(|x|)
$$

as long as $|x| \geq R$ for a continuous nondecreasing radially unbounded function $\alpha_{0}$, a continuously increasing function $\alpha_{1}$, and a positive continuous function $\alpha_{2}$. Then the system (8) is uniformly dissipative in the sense of Levinson.

Various boundedness properties have been considered by Yoshizawa $(1960 ; 1966)$, and their role in establishing existence of self-sustained and forced oscillations has been pointed out. The basic results on dissipativeness in the sense of Levinson are also due to Yoshizawa (1960; 1966).

In the work of Rasvan (2006), some useful results on the study of systems dynamics are given; the author analyzes three different concepts of dissipativeness: classical (standard) dissipativeness, dissipativeness in the sense of Levinson and dissipativeness in the sense of system theory. Criteria, applications and connections between these concepts are proved.

The following result provides conditions on a Lyapunov-Krasovskii functional proposal to guarantee ultimate boundedness of the solutions of a boundary-value problem in finite time. Notice that the result provided below represents nonrestrictive conditions to obtain an 
ultimate bound of a system subject to perturbations or disturbances of the form (6). In fact, for the system under consideration, the additive disturbance variable $\delta(t)$, in general, is assumed to satisfy (7).

Lemma 1 is inspired by the results on input-to-state stability presented by Fridman et al. (2008) (see also Fridman and Dambrine, 2010; Fridman et al., 2010).

Lemma 1. Let $V(\tilde{z}(\cdot, t))$ be a Lyapunov-Krasovskii functional satisfying

$$
\begin{gathered}
\int_{0}^{1} \tilde{z}^{T}(\zeta, t) P \tilde{z}(\zeta, t) \mathrm{d} \zeta \leq V(\tilde{z}(\cdot, t)) \leq \bar{V}(\tilde{z}(\cdot, t)), \\
\frac{\mathrm{d}}{\mathrm{d} t} V(\tilde{z}(\cdot, t))+\sigma V(\tilde{z}(\cdot, t)) \leq \beta \varepsilon \\
\forall t \geq 0, \quad \sigma>0, \quad \beta>0
\end{gathered}
$$

where $P>0, \tilde{z}(\zeta, t)=\left[z_{\zeta}(\zeta, t) \quad z_{t}(\zeta, t) \quad x(t)\right]^{T}$ and $\bar{V}(\tilde{z}(\cdot, t))$ is an upper bound on $V(\tilde{z}(\cdot, t))$. Then, for any initial function $\tilde{\phi}\left(\zeta, t_{0}\right)=\left[\begin{array}{lll}z_{\zeta}\left(\zeta, t_{0}\right) & z_{t}\left(\zeta, t_{0}\right) & x\left(t_{0}\right)\end{array}\right]^{T}$, the ultimate bound defined by

$$
\int_{0}^{1} \tilde{z}^{T}(\zeta, t) P \tilde{z}(\zeta, t) \mathrm{d} \zeta \leq e^{-\sigma t} \bar{V}\left(\tilde{\phi}\left(\zeta, t_{0}\right)\right)+\frac{\beta \varepsilon}{\sigma}
$$

is satisfied for $t_{0}=0, t \geq T_{a}(\tilde{\phi}, \varsigma)>0$, where

$$
T_{a}(\tilde{\phi}, \varsigma)=\frac{1}{\sigma} \ln \left[\frac{1}{\varsigma} \bar{V}\left(\tilde{\phi}\left(\zeta, t_{0}\right)\right)-\frac{\beta \varepsilon}{\varsigma \sigma}\right] .
$$

See the proof in Appendix.

\section{Control design: Practical stabilization conditions}

The main idea in designing stabilizing controllers is to propose a suitable Lyapunov-Krasovskii functional such that the conditions (9) and (10) of Lemma 1 are satisfied along the trajectories of the closed-loop system.

The following result provides a set of conditions under which the trajectories of the drilling system, in a closed-loop with a pair of predetermined controllers, admit an ultimate bound.

Theorem 2. The trajectories of the drilling system described by the coupled wave-ODE system (6) in a closed loop with the controllers

$$
\begin{aligned}
u_{1}(t)= & c_{11} z_{t t}(1, t)+c_{12} z_{t}(1, t) \\
& +c_{13} T_{\mathrm{nl}}\left(z_{t}(1, t)\right)+c_{14} z_{t}(0, t),
\end{aligned}
$$

with $c_{1 i} \in \mathbb{R}^{1 \times 1}, i=1, \ldots, 4$, and

$$
u_{2}(t)=c_{2} x(t), \quad c_{2} \in \mathbb{R}^{1 \times 2},
$$

admit the ultimate bound (11) if the matrix inequalities

$$
P>0, \quad \Psi<0, \quad \Phi<0,
$$

where

$$
\begin{aligned}
& P=\left[\begin{array}{ccc}
a p & \chi[\zeta+1] & 0 \\
\chi[\zeta+1] & p & 0 \\
0 & 0 & R
\end{array}\right] \\
& \Psi=\left[\begin{array}{cc}
-2 p d-\chi+\sigma p & -d \chi[\zeta+1]+\sigma \chi[\zeta+1] \\
* & -a \chi+\sigma p a
\end{array}\right]
\end{aligned}
$$

and

$\Phi$

$$
=\left[\begin{array}{cccccc}
\Phi_{11} & \Phi_{12} & \Phi_{13} & \Phi_{14} & 0 & -2 a \chi h \\
* & \Phi_{22} & \Phi_{23} & \Phi_{24} & E_{1}^{T} R & \Phi_{26} \\
* & * & \Phi_{33} & \Phi_{34} & E_{2}^{T} R & -2 a \chi q \\
* & * & * & \Phi_{44} & 0 & 0 \\
* & * & * & * & \Upsilon_{1}+\sigma R & 0 \\
* & * & * & * & * & 2 a \chi-\beta
\end{array}\right],
$$

with

$$
\begin{aligned}
& \Phi_{11}=2 a \chi h^{2}-a \chi g^{2} c_{11}^{2}, \\
& \Phi_{12}=2 a \chi h k-p a h-a \chi g^{2} c_{11} c_{12}, \\
& \Phi_{13}=2 a \chi h q-a \chi g^{2} c_{11} c_{13}, \\
& \Phi_{14}=a \chi g^{2} c_{11}\left[1-c_{14}\right]+p a g c_{11}, \\
& \Phi_{22}=2 a \chi k^{2}+2 \chi-2 p a k-a \chi g^{2} c_{12}^{2}, \\
& \Phi_{23}=2 a \chi k q-p a q-a \chi g^{2} c_{12} c_{13}, \\
& \Phi_{24}=a \chi g^{2} c_{12}\left[1-c_{14}\right]+p a g c_{12}, \\
& \Phi_{26}=a[p-2 \chi k], \\
& \Phi_{33}=2 a \chi q^{2}-a \chi g^{2} c_{13}^{2}, \\
& \Phi_{34}=a \chi g^{2} c_{13}\left[1-c_{14}\right]+p a g c_{13}, \\
& \Phi_{44}=-a \chi g^{2}\left[1-c_{14}\right]^{2}-\chi-2 p a g+p a g c_{14},
\end{aligned}
$$

are satisfied for some $p>0, \chi>0, \beta>0, R>0$ and any $c_{2} \in \mathbb{R}^{1 \times 2}, c_{1 i} \in \mathbb{R}^{1 \times 1}, i=1, \ldots, 4$.

Proof. Consider the Lyapunov-Krasovskii functional

$$
V(\tilde{z}(\cdot, t))=\int_{0}^{1} \tilde{z}^{T}(\zeta, t) P \tilde{z}(\zeta, t) \mathrm{d} \zeta,
$$

with

$$
\tilde{z}(\zeta, t)=\left[z_{\zeta}(\zeta, t) z_{t}(\zeta, t) x(t)\right]^{T},
$$

and $P$ defined in (14) with $p>0, a>0, \chi>0$ and $R>0$. Notice that condition (9) is satisfied with

$$
\bar{V}(\tilde{z}(\cdot, t))=\lambda_{\max }(P) \int_{0}^{1}\|\tilde{z}(\zeta, t)\|^{2} \mathrm{~d} \zeta .
$$

Now, we establish the conditions under which the inequality (10) is satisfied. The proposed functional can be rewritten as

$$
\begin{aligned}
V\left(z_{\zeta}(\cdot, t), z_{t}(\cdot, t), x_{t}\right)= & V_{1}\left(z_{\zeta}(\zeta, t)\right)+V_{2}\left(z_{t}(\zeta, t)\right) \\
& +V_{3}\left(z_{\zeta}(\zeta, t), z_{t}(\zeta, t)\right)+V_{4}\left(x_{t}\right),
\end{aligned}
$$


where

$$
\begin{aligned}
& V_{1}(\cdot)=p a \int_{0}^{1} z_{\zeta}^{2}(\zeta, t) \mathrm{d} \zeta \\
& V_{2}(\cdot)=p \int_{0}^{1} z_{t}^{2}(\zeta, t) \mathrm{d} \zeta \\
& V_{3}(\cdot)=2 \chi \int_{0}^{1}[\zeta+1] z_{\zeta}(\zeta, t) z_{t}(\zeta, t) \mathrm{d} \zeta \\
& V_{4}(\cdot)=x^{T}(t) R x(t) .
\end{aligned}
$$

Taking time derivatives of each term of $V$, we obtain

$$
\begin{gathered}
\dot{V}_{1}(\cdot)=2 p a \int_{0}^{1} z_{\zeta}(\zeta, t) z_{\zeta t}(\zeta, t) \mathrm{d} \zeta \\
\dot{V}_{2}(\cdot)=2 p \int_{0}^{1} z_{t}(\zeta, t) z_{t t}(\zeta, t) \mathrm{d} \zeta
\end{gathered}
$$

Substituting the wave equation (6a) into (18) yields

$$
\begin{aligned}
\dot{V}_{2}(\cdot)= & 2 p a \int_{0}^{1} z_{t}(\zeta, t) z_{\zeta \zeta}(\zeta, t) \mathrm{d} \zeta \\
& -2 p d \int_{0}^{1} z_{t}^{2}(\zeta, t) \mathrm{d} \zeta .
\end{aligned}
$$

Integration by parts of $\int_{0}^{1} z_{t}(\zeta, t) z_{\zeta \zeta}(\zeta, t) \mathrm{d} \zeta$ gives

$$
\begin{aligned}
\int_{0}^{1} z_{t}(\zeta, t) z_{\zeta \zeta}(\zeta, t) \mathrm{d} \zeta= & \left.z_{t}(\zeta, t) z_{\zeta}(\zeta, t)\right|_{0} ^{1} \\
& -\int_{0}^{1} z_{t \zeta}(\zeta, t) z_{\zeta}(\zeta, t) \mathrm{d} \zeta
\end{aligned}
$$

Then we obtain

$$
\begin{aligned}
\dot{V}_{2}(\cdot)= & \left.2 p a z_{t}(\zeta, t) z_{\zeta}(\zeta, t)\right|_{0} ^{1} \\
& -2 p a \int_{0}^{1} z_{t \zeta}(\zeta, t) z_{\zeta}(\zeta, t) \mathrm{d} \zeta \\
& -2 p d \int_{0}^{1} z_{t}^{2}(\zeta, t) \mathrm{d} \zeta
\end{aligned}
$$

The derivative of $V_{3}$ is

$$
\begin{aligned}
\dot{V}_{3}(\cdot)= & 2 \chi \int_{0}^{1}[\zeta+1] z_{\zeta}(\zeta, t) z_{t t}(\zeta, t) \mathrm{d} \zeta \\
& +2 \chi \int_{0}^{1}[\zeta+1] z_{\zeta t}(\zeta, t) z_{t}(\zeta, t) \mathrm{d} \zeta
\end{aligned}
$$

Substituting the wave equation (6a) yields

$$
\begin{aligned}
\dot{V}_{3}(\cdot)= & 2 a \chi \int_{0}^{1}[\zeta+1] z_{\zeta}(\zeta, t) z_{\zeta \zeta}(\zeta, t) \mathrm{d} \zeta \\
& -2 d \chi \int_{0}^{1}[\zeta+1] z_{\zeta}(\zeta, t) z_{t}(\zeta, t) \mathrm{d} \zeta \\
& +2 \chi \int_{0}^{1}[\zeta+1] z_{\zeta t}(\zeta, t) z_{t}(\zeta, t) \mathrm{d} \zeta
\end{aligned}
$$

Integration by parts of $\int_{0}^{1}[\zeta+1] z_{\zeta}(\zeta, t) z_{\zeta \zeta}(\zeta, t) \mathrm{d} \zeta$ gives

$$
\begin{aligned}
& \int_{0}^{1}[\zeta+1] z_{\zeta}(\zeta, t) z_{\zeta \zeta}(\zeta, t) \mathrm{d} \zeta \\
& =\left.[\zeta+1] z_{\zeta}^{2}(\zeta, t)\right|_{0} ^{1} \\
& \quad-\int_{0}^{1}[\zeta+1] z_{\zeta \zeta}(\zeta, t) z_{\zeta}(\zeta, t) \mathrm{d} \zeta \\
& \quad-\int_{0}^{1} z_{\zeta}^{2}(\zeta, t) \mathrm{d} \zeta
\end{aligned}
$$

Then we obtain

$$
\begin{aligned}
& 2 \int_{0}^{1}[\zeta+1] z_{\zeta}(\zeta, t) z_{\zeta \zeta}(\zeta, t) \mathrm{d} \zeta \\
& \quad=\left.[\zeta+1] z_{\zeta}^{2}(\zeta, t)\right|_{0} ^{1}-\int_{0}^{1} z_{\zeta}^{2}(\zeta, t) \mathrm{d} \zeta
\end{aligned}
$$

Now, observe that

$$
2 z_{\zeta t}(\zeta, t) z_{t}(\zeta, t)=\frac{\partial}{\partial \zeta}\left[z_{t}^{2}(\zeta, t)\right]
$$

Then

$$
\begin{aligned}
2 \int_{0}^{1}[\zeta+1] & z_{\zeta t}(\zeta, t) z_{t}(\zeta, t) \mathrm{d} \zeta \\
& =\int_{0}^{1}[\zeta+1] \frac{\partial}{\partial \zeta}\left[z_{t}^{2}(\zeta, t)\right] \mathrm{d} \zeta
\end{aligned}
$$

Note that $\frac{\partial}{\partial \zeta}\left[z_{t}^{2}(\zeta, t)\right]=2 z_{t}(\zeta, t) z_{\zeta t}(\zeta, t)$. Then

$$
\begin{aligned}
& \int_{0}^{1}[\zeta+1] \frac{\partial}{\partial \zeta}\left[z_{t}^{2}(\zeta, t)\right] \mathrm{d} \zeta \\
& \quad=2 \int_{0}^{1}[\zeta+1] z_{t}(\zeta, t) z_{\zeta t}(\zeta, t) \mathrm{d} \zeta .
\end{aligned}
$$

Integrating by parts, we get

$$
\begin{aligned}
\int_{0}^{1}[\zeta & +1] z_{t}(\zeta, t) z_{\zeta t}(\zeta, t) \mathrm{d} \zeta \\
= & {\left.[\zeta+1] z_{t}^{2}(\zeta, t)\right|_{0} ^{1}-\int_{0}^{1} z_{t}^{2}(\zeta, t) \mathrm{d} \zeta } \\
& -\int_{0}^{1}[\zeta+1] z_{t}(\zeta, t) z_{\zeta t}(\zeta, t) \mathrm{d} \zeta
\end{aligned}
$$

Equivalently,

$$
\begin{aligned}
2 \int_{0}^{1}[\zeta+1] z_{\zeta t}(\zeta, t) z_{t}(\zeta, t) \mathrm{d} \zeta & \\
= & {\left.[\zeta+1] z_{t}^{2}(\zeta, t)\right|_{0} ^{1}-\int_{0}^{1} z_{t}^{2}(\zeta, t) \mathrm{d} \zeta }
\end{aligned}
$$


Substituting (21) and (22) into (20) yields

$$
\begin{aligned}
\dot{V}_{3}(\cdot)= & \left.a \chi[\zeta+1] z_{\zeta}^{2}(\zeta, t)\right|_{0} ^{1}-a \chi \int_{0}^{1} z_{\zeta}^{2}(\zeta, t) \mathrm{d} \zeta \\
& -2 d \chi \int_{0}^{1}[\zeta+1] z_{\zeta}(\zeta, t) z_{t}(\zeta, t) \mathrm{d} \zeta \\
& +\left.\chi[\zeta+1] z_{t}^{2}(\zeta, t)\right|_{0} ^{1}-\chi \int_{0}^{1} z_{t}^{2}(\zeta, t) \mathrm{d} \zeta
\end{aligned}
$$

The derivative of $V_{4}$ along the trajectories of (6d) is then

$$
\begin{aligned}
\dot{V}_{4}\left(x_{t}\right)= & \dot{x}^{T}(t) R x(t)+x^{T}(t) R \dot{x}(t) \\
= & {\left[A x(t)+B u_{2}(t)+E_{1} z_{t}(1, t)\right.} \\
& \left.+E_{2} T_{\mathrm{nl}}\left(z_{t}(1, t)\right)\right]^{T} R x(t) \\
& +x^{T}(t) R\left[A x(t)+B u_{2}(t)\right. \\
& \left.+E_{1} z_{t}(1, t)+E_{2} T_{\mathrm{nl}}\left(z_{t}(1, t)\right)\right] .
\end{aligned}
$$

We seek the stabilization of axial drilling dynamics. For the sake of simplicity, we choose $u_{2}$ as in (13). This choice guarantees the quadratic form of $\dot{V}_{4}\left(x_{t}\right)$, i.e.,

$$
\dot{V}_{4}\left(x_{t}\right)=x^{T}(t) \Upsilon_{1} x(t)+\Upsilon_{2}^{T} R x(t)+x^{T}(t) R \Upsilon_{2},
$$

where

$$
\begin{aligned}
& \Upsilon_{1}=A^{T} R+R A+c_{2}^{T} B^{T} R+R B c_{2}, \\
& \Upsilon_{2}=E_{1} z_{t}(1, t)+E_{2} T_{\mathrm{nl}}\left(z_{t}(1, t)\right) .
\end{aligned}
$$

In view of (17), 19, 23) and (24), the time derivative of $V$ is given by

$$
\begin{aligned}
\dot{V}(\cdot)= & 2 p a \int_{0}^{1} z_{\zeta}(\zeta, t) z_{\zeta t}(\zeta, t) \mathrm{d} \zeta \\
& -2 p a \int_{0}^{1} z_{t \zeta}(\zeta, t) z_{\zeta}(\zeta, t) \mathrm{d} \zeta \\
& -2 p d \int_{0}^{1} z_{t}^{2}(\zeta, t) \mathrm{d} \zeta-a \chi \int_{0}^{1} z_{\zeta}^{2}(\zeta, t) \mathrm{d} \zeta \\
& -2 d \chi \int_{0}^{1}[\zeta+1] z_{\zeta}(\zeta, t) z_{t}(\zeta, t) \mathrm{d} \zeta \\
& -\chi \int_{0}^{1} z_{t}^{2}(\zeta, t) \mathrm{d} \zeta+\left.2 p a z_{t}(\zeta, t) z_{\zeta}(\zeta, t)\right|_{0} ^{1} \\
& +\left.a \chi[\zeta+1] z_{\zeta}^{2}(\zeta, t)\right|_{0} ^{1} \\
& +\left.\chi[\zeta+1] z_{t}^{2}(\zeta, t)\right|_{0} ^{1} \\
& +x^{T}(t) \Upsilon_{1} x(t)+\Upsilon_{2}^{T} R x(t)+x^{T}(t) R \Upsilon_{2}
\end{aligned}
$$

In view of the second condition of Lemma 1, we derive the following expression:

$$
\begin{aligned}
& \dot{V}(\cdot)+\sigma V(\cdot) \\
& =-2 p d \int_{0}^{1} z_{t}^{2}(\zeta, t) \mathrm{d} \zeta-a \chi \int_{0}^{1} z_{\zeta}^{2}(\zeta, t) \mathrm{d} \zeta \\
& \quad-2 d \chi \int_{0}^{1}[\zeta+1] z_{\zeta}(\zeta, t) z_{t}(\zeta, t) \mathrm{d} \zeta \\
& \quad-\chi \int_{0}^{1} z_{t}^{2}(\zeta, t) \mathrm{d} \zeta+x^{T}(t) \Upsilon_{1} x(t) \\
& \quad+\Upsilon_{2}^{T} R x(t)+x^{T}(t) R \Upsilon_{2}+\sigma p a \int_{0}^{1} z_{\zeta}^{2}(\zeta, t) \mathrm{d} \zeta \\
& \quad+\sigma p \int_{0}^{1} z_{t}^{2}(\zeta, t) \mathrm{d} \zeta \\
& +2 \sigma \chi \int_{0}^{1}[\zeta+1] z_{\zeta}(\zeta, t) z_{t}(\zeta, t) \mathrm{d} \zeta \\
& +\sigma x^{T}(t) R x(t)+\Pi,
\end{aligned}
$$

where

$$
\begin{aligned}
\Pi= & \left.2 p a z_{t}(\zeta, t) z_{\zeta}(\zeta, t)\right|_{0} ^{1}+\left.a \chi[\zeta+1] z_{\zeta}^{2}(\zeta, t)\right|_{0} ^{1} \\
& +\left.\chi[\zeta+1] z_{t}^{2}(\zeta, t)\right|_{0} ^{1} \\
= & 2 \chi z_{t}^{2}(1, t)+2 a \chi z_{\zeta}^{2}(1, t)+2 p a z_{t}(1, t) z_{\zeta}(1, t) \\
& -\chi z_{t}^{2}(0, t)-a \chi z_{\zeta}^{2}(0, t)-2 p a z_{t}(0, t) z_{\zeta}(0, t) .
\end{aligned}
$$

The introduction of the boundary conditions (6b)-6c yields

$$
\begin{aligned}
\Pi= & 2 \chi z_{t}^{2}(1, t)+2 a \chi\left[-h z_{t t}(1, t)-k z_{t}(1, t)\right. \\
& \left.-q T_{\mathrm{nl}}\left(z_{t}(1, t)\right)+\delta(t)\right]^{2} \\
& +2 p a z_{t}(1, t)\left[-h z_{t t}(1, t)-k z_{t}(1, t)\right. \\
& \left.-q T_{\mathrm{nl}}\left(z_{t}(1, t)\right)+\delta(t)\right] \\
& -\chi z_{t}^{2}(0, t)-a \chi\left[g\left[z_{t}(0, t)-u_{1}(t)\right]\right]^{2} \\
& -2 p a z_{t}(0, t)\left(g\left(z_{t}(0, t)-u_{1}(t)\right)\right) .
\end{aligned}
$$

Our purpose is to rearrange the terms of 25) into symmetric matrices. According to the condition (10), the negative definiteness of these matrices guarantees ultimate boundedness of the system trajectories. The choice of the controller $u_{1}$ is based on the fact that a necessary condition for a symmetric matrix to be negative definite is that all the diagonal entries be negative.

For the stabilization of torsional trajectories we propose the controller structure given in 12, which facilitates the possibility of the negative definiteness of the obtained symmetric matrices. More precisely, $\Pi$ is written 
as

$$
\begin{aligned}
\Pi= & 2 a \chi\left[h^{2} z_{t t}^{2}(1, t)+2 h k z_{t}(1, t) z_{t t}(1, t)\right. \\
& +2 h q z_{t t}(1, t) T_{\mathrm{nl}}\left(z_{t}(1, t)\right)-2 h z_{t t}(1, t) \delta(t) \\
& +k^{2} z_{t}^{2}(1, t)+2 k q z_{t}(1, t) T_{\mathrm{nl}}\left(z_{t}(1, t)\right) \\
& -2 k z_{t}(1, t) \delta(t)+q^{2} T_{\mathrm{nl}}^{2}\left(z_{t}(1, t)\right) \\
& \left.-2 q T_{\mathrm{nl}}\left(z_{t}(1, t)\right) \delta(t)+\delta(t)^{2}\right]+2 \chi z_{t}^{2}(1, t) \\
& +2 p a z_{t}(1, t)\left[-h z_{t t}(1, t)-k z_{t}(1, t)\right. \\
& \left.-q T_{\mathrm{nl}}\left(z_{t}(1, t)\right)+\delta(t)\right]-a \chi g^{2}\left[c_{11}^{2} z_{t t}^{2}(1, t)\right. \\
& +2 c_{11} c_{12} z_{t}(1, t) z_{t t}(1, t)+c_{12}^{2} z_{t}^{2}(1, t) \\
& +2 c_{11} c_{13} z_{t t}(1, t) T_{\mathrm{nl}}\left(z_{t}(1, t)\right) \\
& -2 c_{11}\left[1-c_{14}\right] z_{t t}(1, t) z_{t}(0, t) \\
& +2 c_{12} c_{13} z_{t}(1, t) T_{\mathrm{nl}}\left(z_{t}(1, t)\right) \\
& -2 c_{12}\left[1-c_{14}\right] z_{t}(1, t) z_{t}(0, t) \\
& +c_{13}^{2} T_{\mathrm{nl}}^{2}\left(z_{t}(1, t)\right)+\left[1-c_{14}\right]^{2} z_{t}^{2}(0, t) \\
& \left.-2 c_{13}\left[1-c_{14}\right] T_{\mathrm{nl}}\left(z_{t}(1, t)\right) z_{t}(0, t)\right] \\
& -\chi z_{t}^{2}(0, t)-2 p a g z_{t}(0, t)\left[z_{t}(0, t)\right. \\
& -c_{11} z_{t t}(1, t)-c_{12} z_{t}(1, t) \\
& \left.-c_{13} T_{\mathrm{nl}}\left(z_{t}(1, t)\right)-c_{14} z_{t}(0, t)\right] .
\end{aligned}
$$

From the inequality (7) we have that $\delta(t)^{2}-\varepsilon \leq 0$. Then, for any $\beta>0$, the following inequality is satisfied:

$$
-\beta\left[\delta(t)^{2}-\varepsilon\right] \geq 0 .
$$

This condition represents an additional restriction to be considered. To this end, we take it into consideration within the stabilizing condition given by the second condition of Lemma 1 i.e., we add the term (26) to the expression for $\dot{V}(\cdot)+\sigma V(\cdot)$ and we obtain an inequality of the form $\dot{V}(\cdot)+\sigma V(\cdot)-\beta \varepsilon \leq \Xi$, where $\Xi$ is a quadratic term, defined according to Eqns. 25) (26). After symmetrization of the cross terms, we have that $\Xi=\int_{0}^{1} \eta_{1}^{T} \Psi \eta_{1} d \zeta+\eta_{2}^{T} \Phi \eta_{2}$. Then

$$
\frac{\mathrm{d}}{\mathrm{d} t} V(\cdot)+\sigma V(\cdot)-\beta \varepsilon \leq \int_{0}^{1} \eta_{1}^{T} \Psi \eta_{1} \mathrm{~d} \zeta+\eta_{2}^{T} \Phi \eta_{2},
$$

where

$$
\begin{aligned}
& \eta_{1}=\left[z_{t}(\zeta, t) z_{\zeta}(\zeta, t)\right]^{T}, \\
& \eta_{2}=\left[z_{t t}(1, t) z_{t}(1, t) T_{\mathrm{nl}}\left(z_{t}(1, t)\right) z_{t}(0, t) x(t) \delta(t)\right]^{T},
\end{aligned}
$$

and $\Psi, \Phi$ are defined in (15) and (16), respectively.

The fulfillment of the inequality (10) is conditioned by the negative definiteness of matrices $\Psi$ and $\Phi$, which entails the problem of the feasibility of the LMI $\Psi<0$ and the BMI $\Phi<0$. Notice that the particular structure of the controller $u_{1}$ with appropriate values of $c_{1 i}$ ensures the negativity of the diagonal terms of matrix $\Phi$.
Remark 1. The choice of the term $\chi[\zeta+1]$ in 14 does not contribute to the positive definiteness of matrix $P$. In fact, taking $\chi \zeta$ instead of $\chi[\zeta+1]$ leads to less conservative conditions to satisfy $P>0$ (considering $\chi \zeta$ instead of $\chi[\zeta+1]$ in (14), $P>0$ is always satisfied for $\zeta=0$, and for $\zeta=1$, the inequality is guaranteed for $a p^{2}>\chi^{2}$, but considering $\chi[\zeta+1]$, the positive definiteness of $P$ is restricted to $a p^{2}>\chi^{2}$ for $\zeta=0$, and to $a p^{2}>4 \chi^{2}$ for $\zeta=1$ ). However, if we consider $\chi \zeta$ instead of $\chi[\zeta+1]$ in (14), matrix $\Phi$ would never be negative definite since the terms $-a \chi g^{2} c_{11}^{2}$ and $-a \chi g^{2} c_{13}^{2}$ would not appear and the diagonal terms $\Phi_{11}$ and $\Phi_{33}$ would be positive.

\section{Numerical results}

In this section, the effectiveness of the proposed control approach is highlighted through simulations results.

The numerical values of the physical parameters used in the following simulations (given in Table 1) reflect typical operating conditions in real oilwell drilling platforms.

Table 1. Numerical values of the drilling system parameters

\begin{tabular}{|c|c|c|c|}
\hline Symbol & Value & Symbol & Value \\
\hline \hline$G$ & $79.3 \times 10^{9} \mathrm{~N} \mathrm{~m}^{-2}$ & $I$ & $0.095 \mathrm{~kg} \mathrm{~m}$ \\
$J$ & $1.19 \times 10^{-5} \mathrm{~m}^{4}$ & $R_{\mathrm{b}}$ & $0.155575 \mathrm{~m}$ \\
$W_{\mathrm{ob}}$ & $97347 \mathrm{~N}$ & $I_{\mathrm{B}}$ & $89 \mathrm{~kg} \mathrm{~m}^{2}$ \\
$\mu_{\mathrm{cb}}$ & 0.5 & $\mu_{\mathrm{sb}}$ & 0.8 \\
$c_{\mathrm{b}}$ & $0.03 \mathrm{~N} \mathrm{~m} \mathrm{sad}^{-1}$ & $m_{0}$ & $37278 \mathrm{~kg}$ \\
$k_{0}$ & $1.55 \times 10^{6} \mathrm{~kg} \mathrm{~s}^{-2}$ & $\Gamma_{0}$ & $0.1 \mathrm{~m} \mathrm{~s}^{-1}$ \\
$L$ & $1172 \mathrm{~m}$ & $c_{0}$ & $16100 \mathrm{~kg} \mathrm{~s}^{-1}$ \\
$\mu_{1}$ & $257 \mathrm{~m}^{-1}$ & $\gamma_{\mathrm{b}}$ & 0.9 \\
$c_{\mathrm{a}}$ & $2000 \mathrm{~N} \mathrm{~m} \mathrm{~s}$ & $\varsigma$ & 0.1 \\
$\varepsilon$ & 0.5 & $\gamma$ & $0.1 \mathrm{~N} \mathrm{~s}$ \\
\hline
\end{tabular}

The numerical values corresponding to the torsional and axial drilling dynamics were taken from the work of Challamel (2000), and the ones corresponding to the frictional torque on the bit model from that of Navarro-López and Suárez (2004).

The dry friction torque at the rock-bit interface is approximated by the nonlinear function given in (5). It is assumed that the additive noise signal accounting for external disturbances and modeling errors satisfies $\delta(t)^{2} \leq 0.5$. The initial conditions are such that

$$
\int_{0}^{1}\left\|\tilde{\phi}\left(\zeta, t_{0}\right)\right\|^{2} \mathrm{~d} \zeta=3.25 .
$$

The torsional and axial reference velocities considered are $10 \mathrm{rad} \mathrm{s}^{-1}$ and $0.1 \mathrm{~ms}^{-1}$, respectively.

5.1. Steady-state, stick-slip and bit-bounce. The stick-slip phenomenon consisting in torsional vibration of 
the drillstring constitutes the major source of failures in drilling operations. It is characterized by stick phases, during which the rotation stops completely, and slip phases, during which the angular velocity of the tool increases up to two times the nominal angular velocity. This phenomenon occurs when a section of the rotating drillstring is momentarily caught by friction against the borehole, and then releases. The bit might eventually get stuck and then, after accumulating energy in terms of torsion, be suddenly released; the string rotation speeds up dramatically and large centrifugal accelerations occur.

The occurrence of torsional vibrations contributes to the excitation of irregular movements of the drilling components along their longitudinal axis causing bit-bounce and rough drilling behavior that destroys the drill bit, damages the BHA and increases the total drilling time.

The steady-state of the drilling system corresponds to a constant rate of penetration of the drill bit $\Gamma$ while rotating at a constant speed $\Omega$. Figure 2 shows the angular and axial bit velocities in the steady-state. Simulation results are in close agreement with field observations regarding the stick-slip and the bit-bounce.
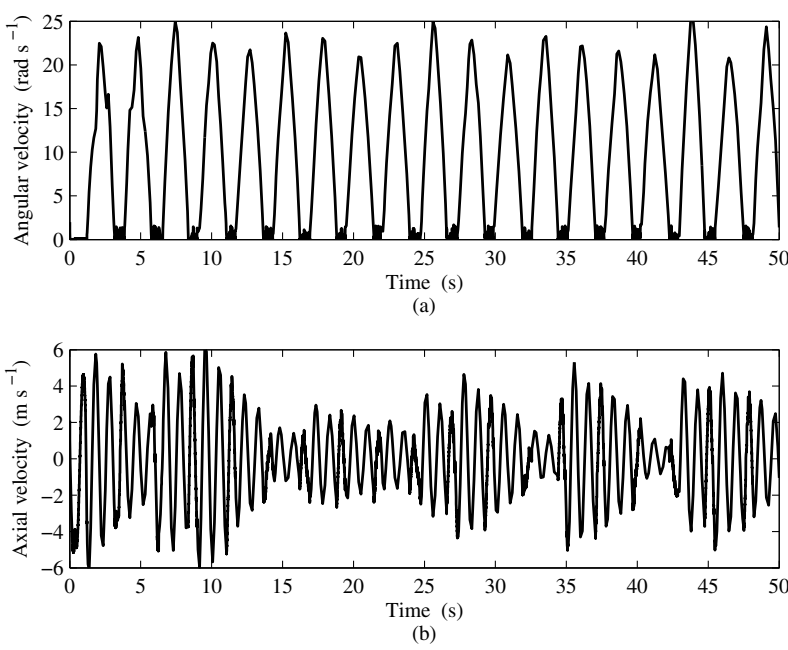

Fig. 2. Drilling system trajectories in the steady-state: bit angular velocity $z_{t}(1, t)$ (stick-slip) (a), bit axial velocity $\dot{Y}(t)$ (bit-bounce) (b).

The steady-state performance undergoes various qualitative changes when the rotating and penetration speeds are changed. These qualitative changes are typically captured in a bifurcation diagram that characterizes the variations of equilibrium points into limit cycling vibrations. Figure 3 shows the torsional trajectories of the drilling system in a two-dimensional phase with the relative variable $z_{t}(1, t)$; the bit motion converges to a limit cycle.

In the work of Boussaada et al. (2012), the stability

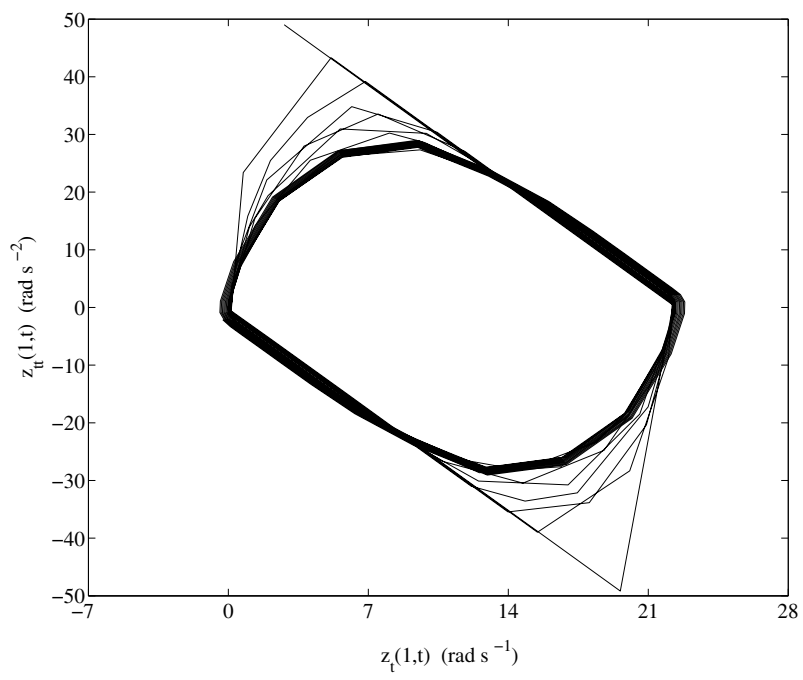

Fig. 3. Stick-slip limit cycle.

of the steady-state of the drilling system (considering coupled axial-torsional dynamics) is investigated; the qualitative dynamic response of the system is characterized through a local bifurcation analysis. With this study, it is concluded that large speeds are eventually stable for all weights on the bit, but such large speeds may not be practically feasible. In fact, a common empirical strategy to avoid torsional drilling vibrations consists in increasing the angular velocity provided by the rotary table. One of the disadvantages of the strategy is that a substantial increment in the angular speed induces lateral problems, such as irregular rotation, which causes repeated collisions between the rod and the borehole walls. The shocks may damage the drilling system components and deteriorate the borehole wall affecting the overall drilling direction. Even though operational guidelines are helpful in reducing string vibrations, they cannot guarantee an optimal drilling operation; for this reason, effective elimination of drilling vibrations requires feedback control actions.

5.2. Control of drilling vibrations. The proposed control approach ensures ultimate boundedness of a measure involving torsional-axial drilling system trajectories; the strategy succeeds in eliminating the stick-slip and bit-bounce phenomena.

Using Theorem 2, it is possible to synthesize the controllers (13) and (12) by means of an appropriate computational tool such as the add-on toolbox PENBMI integrated with the TOMLAB optimization environment. This toolbox, developed in cooperation with PENOPT GbR as well as researchers Michal Kǒcvara and Michael Stingl of Erlangen-Nürnberg University, solves convex and semi-definite programming problems subject to linear 
and bilinear matrix inequalities. The algorithm, developed on MATLAB and $\mathrm{C}$ code, combines ideas of the (exterior) penalty and (interior) barrier methods (Ben-Tal and Zibulevsky, 1997) with the augmented Lagrangian method. Roughly speaking, the method is based on the selection of penalty/barrier functions penalizing inequality constraints and satisfying some properties such as strict convexity, by instance. For a detailed description of the algorithm, see the work of Kǒcvara and Stingl (2003).

The maximum exponential decay rate for which the conditions of Theorem 2 are satisfied is $\sigma=0.8$. A feasible result of the LMI $\Psi<0$ with $\Psi$ given in (15) is

$$
p=0.7406, \quad \chi=0.9559 .
$$

Using the above values, a feasible result of the BMI $\Phi<0$ with $\Phi$ given in (16) is

$$
\begin{aligned}
c_{11} & =-0.0067, & c_{12} & =-0.04699, \\
c_{14} & =0.6642, & c_{13} & =-0.0548, \\
c_{2} & =\left[\begin{array}{ll}
0 & 2.0234
\end{array}\right], & \beta & =16.8149,
\end{aligned}
$$

$$
R=\left[\begin{array}{cc}
551.7373 & 11.7383 \\
11.7383 & 13.4465
\end{array}\right]
$$

The stabilizing controllers are then given by

$$
\begin{aligned}
u_{1}(t)= & -0.0067 z_{t t}(1, t)-0.04699 z_{t}(1, t) \\
& -0.0548 T_{\mathrm{nl}}\left(z_{t}(1, t)\right)+0.6642 z_{t}(0, t),
\end{aligned}
$$

and

$$
u_{2}(t)=2.0234 \dot{y}(t)=2.0234 \dot{Y}(t)-2.0234 \Gamma_{0} .
$$

Figure 4 shows the trajectories of the controllers $u_{1}$ and $u_{2}$; note that they do not exhibit significant variations, which is a favorable feature regarding the saturation constraints of control inputs in real plants.

Figures 5 and 6 show the closed-loop system trajectories $z_{t}(\zeta, t)$ (distributed angular velocity), $x_{1}=$ $y=Y-\Gamma_{0} t$ (deviated axial position) and $x_{2}=$ $\dot{y}=\dot{Y}-\Gamma_{0}$ (deviated axial velocity). According to the obtained results, the stick-slip and bit-bounce are effectively eliminated by means of the wave-ODE model-based controllers (28)-(29).

Notice that, initially, small amplitude oscillations are observed at the surface $(\zeta=0)$, while at the bottom $(\zeta=$ $1)$ the amplitude of the oscillations is more important, as reported in real wells.

In view of Lemma 1, an ultimate bound on the drilling system trajectories is given by

$$
\begin{aligned}
\int_{0}^{1} \tilde{z}^{T}(\zeta, t) P \tilde{z}(\zeta, t) \mathrm{d} \zeta & \leq e^{-\sigma t} \bar{V}\left(\tilde{\phi}\left(\zeta, t_{0}\right)\right)+\frac{\beta \varepsilon}{\sigma} \\
& =1.79 \times 10^{3} e^{-0.8 t}+10.5093
\end{aligned}
$$
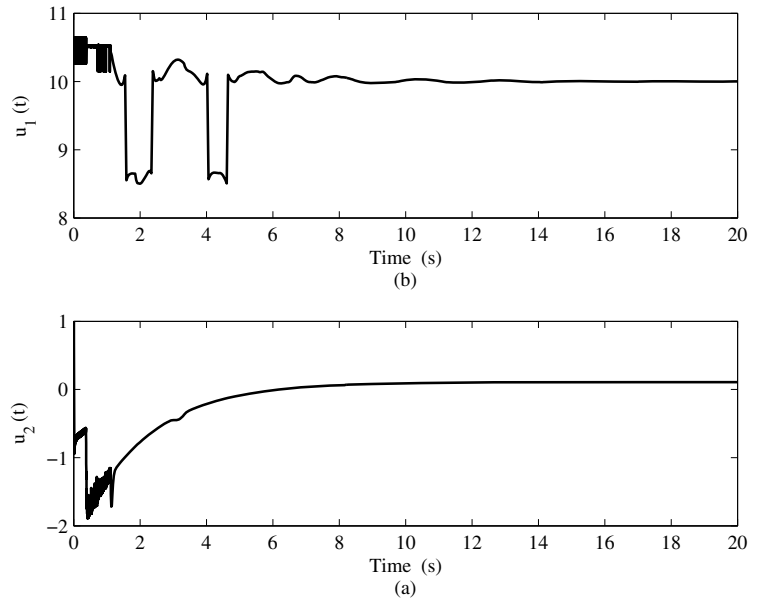

Fig. 4. Control trajectories.

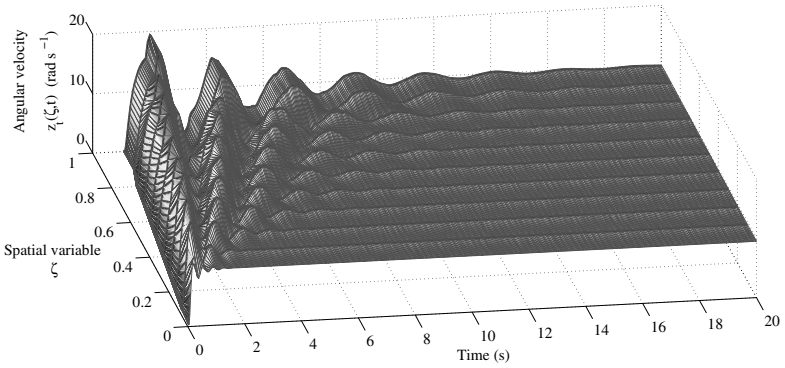

Fig. 5. Distributed angular velocity $z_{t}(\zeta, t), \zeta \in(0,1)$; the closed-loop system [6, 28)-29].

where

$$
\bar{V}\left(\tilde{\phi}\left(\zeta, t_{0}\right)\right)=\lambda_{\max }(P) \int_{0}^{1}\left\|\tilde{\phi}\left(\zeta, t_{0}\right)\right\|^{2} \mathrm{~d} \zeta
$$

for $t \geq T_{a}(\tilde{\phi}, \varsigma)>0$,

$$
\begin{aligned}
T_{a}(\tilde{\phi}, \varsigma) & =\frac{1}{\sigma} \ln \left[\frac{1}{\varsigma} \lambda_{\max }(P) \int_{0}^{1}\|\tilde{\phi}(\zeta, t)\|^{2} \mathrm{~d} \zeta-\frac{\beta \varepsilon}{\varsigma \sigma}\right] \\
& =12.2361 \mathrm{~s} .
\end{aligned}
$$

In order to assess the performance of the wave-ODE model-based control approach, the next section presents a brief review of two additional control strategies to eliminate drilling vibrations: the soft-torque controller and the flatness-based control method. The response of the system under the three different control strategies is compared.

5.3. Discussion. As explained above, there exist several methods to tackle drilling vibrations; this section provides a brief review of two of them: the soft-torque system and the flatness-based control approach. Simulations results show that both of them lead to the suppression of torsional and axial vibrations; 

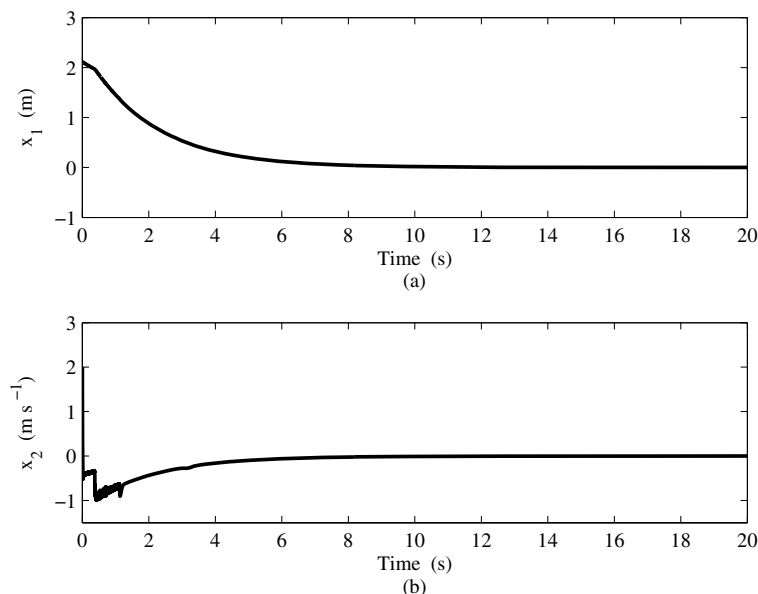

Fig. 6. Axial trajectories of the closed loop system (6), (28)29]: $x_{1}=Y-\Gamma_{0} t$ (a), $x_{2}=\dot{Y}-\Gamma_{0}$ (b). Variables $Y$ and $\dot{Y}$ represent the axial position and axial velocity, respectively.

however, improved results can be obtained by using the control approach proposed in this contribution, compared with the response observed when the specific controllers presented by Tucker and Wang (1999) as well as Saldivar et al. (2014) are used.

Soft torque rotary system (STRS). This system was developed by the Shell company in the early 1990s (Javanmardi and Gaspard, 1992). The STRS is a PI-like speed controller which involves a pair of gains $\kappa_{i}$ (drive stiffness in $\mathrm{Nm} / \mathrm{rad}$ ) and $\kappa_{p}$ (drive damping in $\mathrm{Nms} \mathrm{rad}^{-1}$ ) that must be calculated according to the drillstring and BHA configuration. The STRS is defined as follows (Tucker and Wang, 1999):

$$
\left\{\begin{aligned}
u_{\Omega}(t) & =\kappa_{p} \dot{\tilde{\xi}}(t)+\kappa_{i} \tilde{\xi}(t), \\
\tilde{\xi}(t) & =\Omega_{0} t-\frac{\nu}{\omega_{c}} T_{c}(t)-z(0, t)+\xi_{0},
\end{aligned}\right.
$$

where $u_{\Omega}(t)$ is the motor torque provided by the rotary table, $\Omega_{0}$ denotes a prescribed angular velocity, $\xi_{0}$ is the displacement of the drillstring at the upper extremity from its reference value, $\omega_{c}$ is the cut-off angular frequency and $\nu$ is an additional control parameter. The variable $z(0, t)$ denotes the angular position at the top extremity, $\kappa_{p}$ and $\kappa_{i}$ are the proportional and integral controller gains, and $T_{c}$ is the output of a low-pass filter applied to the contact torque.

Flatness-based control. In nonlinear systems theory, the flatness property refers to the ability of dynamical systems of being exactly linearized via endogenous feedback (Fliess et al., 1995). The main attribute of flat systems is that the state and input variables can be directly expressed without integrating any differential equation, in terms of one particular set of variables called a flat output (or a linearizing output) and a finite number of its derivatives
(Fliess et al., 1995). The flatness property of a nonlinear dynamical system is useful to deal with trajectory tracking problems. The flatness property of the drilling system was proved by Knuppel et al. (2014). Based on the fact that the elimination of drilling vibrations requires the angular and axial velocities of the drilling bit to follow a constant reference path, a pair of controllers aimed at tackling the steering problem is designed by Saldivar et al. (2014).

The flatness-based control laws are defined as

$$
\left\{\begin{aligned}
u_{\Omega}(t)= & k_{1} z_{t}(0, t)+k_{2}\left[z_{t}(1, t-\tau)+\gamma(t)\right] \\
& +k_{3} v(t)+k_{4} T_{\mathrm{nl}}\left(z_{t}(1, t-\tau)+\gamma(t)\right) \\
v(t)= & k_{5} z_{t t}^{\mathrm{ref}}(1, t+\tau)+k_{6} I+k_{7} z_{t t}(1, t-\tau) \\
& +k_{8} T_{\mathrm{nl}}\left(z_{t}(1, t-\tau)+\gamma(t)\right) \\
& -k_{8} T_{\mathrm{nl}}\left(z_{t}(1, t-\tau)\right) \\
I= & 2 z_{t}(0, t)-z_{t}(1, t-\tau)-z_{t}^{\mathrm{ref}}(1, t+\tau), \\
\gamma(t)= & \int_{t-2 \tau}^{t} v(\xi) \mathrm{d} \xi
\end{aligned}\right.
$$

and

$$
\left\{\begin{aligned}
u_{\Gamma}(t)= & \bar{k}_{1} w_{t}(0, t)+\bar{k}_{2}\left[w_{t}(1, t-\tilde{\tau})+\bar{\gamma}(t)\right] \\
& +\bar{k}_{3} \bar{v}(t)+\bar{k}_{4} T_{\mathrm{nl}}\left(z_{t}(1, t-\tau)+\gamma(t)\right) \\
\bar{v}(t)= & \bar{k}_{5} w_{t t}^{\mathrm{ref}}(1, t+\tilde{\tau})-\bar{k}_{6} \bar{I}+\bar{k}_{7} w_{t t}(1, t-\tilde{\tau}) \\
& +\bar{k}_{8} T_{\mathrm{nl}}\left(z_{t}(1, t-\tau)+\gamma(t)\right) \\
& -\bar{k}_{8} T_{\mathrm{nl}}\left(z_{t}(1, t-\tau)\right) \\
\bar{I}= & 2 w_{t}(0, t)-w_{t}(1, t-\tilde{\tau})-w_{t}^{\mathrm{ref}}(1, t+\tilde{\tau}), \\
\bar{\gamma}(t)= & \int_{t-2 \tilde{\tau}}^{t} \bar{v}(\xi) \mathrm{d} \xi
\end{aligned}\right.
$$

where $z_{t}$ and $w_{t}$ denote the angular and axial drillstring velocities, respectively, $u_{\Omega}(t)$ and $u_{\Gamma}(t)$ stand for the torque provided by the rotor at the surface and the upward hook force, and they satisfy the relations $u_{\Omega}(t)=\bar{\alpha} u_{1}(t)$, $u_{\Gamma}(t)=\bar{\beta} u_{2}(t)$, where the angular momentum and the viscous friction coefficient are taken as $\bar{\alpha}=2000 \mathrm{~N} \mathrm{~m} \mathrm{~s}$ $\operatorname{rad}^{-1}$ and $\bar{\beta}=200.025 \mathrm{~kg} \mathrm{~s}^{-1}$, respectively. The time delays $\tau, \tilde{\tau}$ and the controller gains $k_{i}, \bar{k}_{i}, i=1, \ldots, 8$, depend on the physical parameters of the system.

The main idea of flatness-based control design is to compute the control inputs such that the errors between the desired and actual trajectories satisfy the stable dynamics leading to exponential convergence of the torsional and axial trajectories $z_{t}(1, t), w_{t}(1, t)$ to the reference velocities $z_{t}^{\text {ref }}(1, t), w_{t}^{\text {ref }}(1, t)$. Notice that the advanced terms in (31) and (32) correspond to prescribed references, computed at $t+\tau$ and at $t+\tilde{\tau}$, i.e., in advance of time.

Figure 7 shows the angular and axial bit velocities of the drilling system in a closed loop with three control strategies: the soft-torque system, flatness-based controllers and the control method proposed in this 
contribution. Clearly, the system trajectories converge faster to the desired target via the control laws 28) -29 .

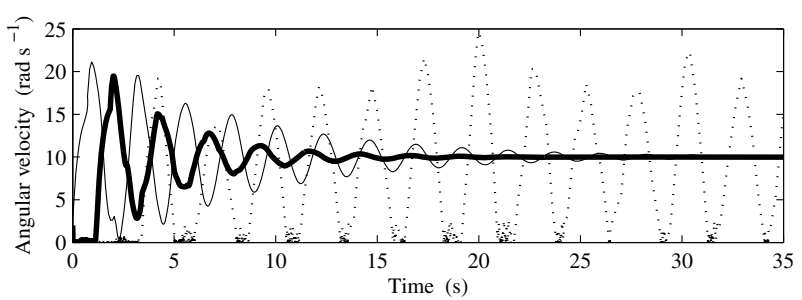

(a)

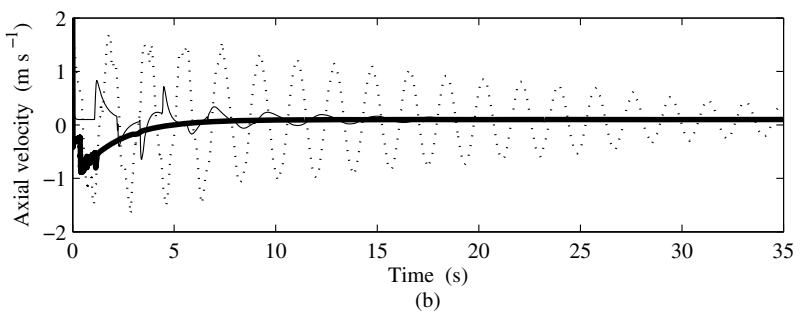

Fig. 7. Bit trajectories under different control strategies-the soft-torque control (30) (dotted line), the flatness-based controllers (31)-32) (thin line), the wave-ODE modelbased controllers 28, -29) (thick line): angular velocity at the bottom extremity $z_{t}(1, t)$ (a), axial velocity at the bottom extremity $\dot{Y}(t)=w_{t}(1, t)(\mathrm{b})$.

Note that although the STRS is aimed at eliminating only the stick-slip phenomenon, the axial vibrations are also diminished because of the coupling between torsional and longitudinal dynamics.

Numerical values of the gains of the soft torque and flatness-based controllers used by Tucker and Wang (1999) as well as Saldivar et al. (2014) are given in Table 2

\section{Concluding remarks}

We have addressed the problem of control design for a class of coupled wave-ODE systems; the importance of the present contribution lies in the significant engineering application involved: the elimination of drilling vibrations.

Through LMI-BMI techniques we have established feedback controllers guaranteeing ultimate boundedness of the system trajectories, which inherently implies practical stabilization of the coupled system.

The performance of the proposed strategy is evidenced by simulations of the drilling system under three different control approaches: the soft-torque system, flatness-based control and the one proposed here. It has been shown that the control method presented in this contribution provides improved results; the stick-slip and bit-bounce are eliminated faster than with the other control solutions.
Table 2. Numerical values of the gains of the soft torque and flatness-based controllers.

\begin{tabular}{|c|c|}
\hline Control gain & Numerical value \\
\hline \hline$k_{p}$ & $0.3658 \mathrm{~N} \mathrm{~m} \mathrm{~s} \mathrm{rad}^{-1}$ \\
$k_{1}$ & $2.0048 \times 10^{3} \mathrm{~N} \mathrm{~m} \mathrm{~s} \mathrm{rad}^{-1}$ \\
$k_{2}$ & $-4.8067 \mathrm{~N} \mathrm{~m} \mathrm{~s} \mathrm{rad}^{-1}$ \\
$k_{3}$ & $30000 \mathrm{~N} \mathrm{~m} \mathrm{~s}^{2} \mathrm{rad}^{-1}$ \\
$k_{4}$ & 210 \\
$k_{5}$ & $6.4085 \times 10^{-5}$ \\
$k_{6}$ & $-1.6021 \times 10^{-4} \mathrm{~s}^{-1}$ \\
$k_{7}$ & 0.9999 \\
$k_{8}$ & $-0.0070 \mathrm{rad} \mathrm{N}^{-1} \mathrm{~m}^{-1} \mathrm{~s}^{-2}$ \\
$k_{i}$ & $0.1672 \mathrm{~N} \mathrm{~m} \mathrm{rad}^{-1}$ \\
$\bar{k}_{1}$ & $1.4020 \times 10^{5} \mathrm{~N} \mathrm{~s} \mathrm{~m}^{-1}$ \\
$\bar{k}_{2}$ & $-140000 \mathrm{~N} \mathrm{~s} \mathrm{~m}^{-1}$ \\
$\bar{k}_{3}$ & $40000 \mathrm{~kg}^{-1}$ \\
$\bar{k}_{4}$ & $3500 \mathrm{~m}^{-1}$ \\
$\bar{k}_{5}$ & 0.5833 \\
$\bar{k}_{6}$ & $-1.4583 \mathrm{~s}^{-1}$ \\
$\bar{k}_{7}$ & 0.4167 \\
$\bar{k}_{8}$ & $-0.0365 \mathrm{~N}^{-1} \mathrm{~s}^{-2}$ \\
\hline
\end{tabular}

It is worth mentioning that the proposed systematic method to design stabilizing control laws can be easily extended to treat more general systems.

\section{Acknowledgment}

Belem Saldivar is a CONACYT research fellow.

\section{References}

Anabtawiii, M. (2011). Practical stability of nonlinear stochastic hybrid parabolic systems of Itô-type: Vector Lyapunov functions approach, Nonlinear Analysis: Real World Applications 12(1): 1386-1400.

Bailey, J. and Finnie, I. (1960). An analytical study of drillstring vibration, Journal of Engineering for Industry, Transactions of the ASME 82(2): 122-128.

Ben-Tal, A. and Zibulevsky, M. (1997). Penalty/barrier multiplier methods for convex programming problems, SIAM Journal on Optimization 7(2): 347-366.

Boussaada, I., Mounier, H., Niculescu, S. and Cela, A. (2012). Analysis of drilling vibrations: A time delay system approach, 20th Mediterranean Conference on Control and Automation MED, Barcelona, Spain, pp. 610-614.

Canudas-de Wit, C., Rubio, F. and Corchero, M. (2008). D-OSKIL: A new mechanism for controlling stick-slip oscillations in oil well drillstrings, IEEE Transactions on Control Systems Technology 16(6): 1177-1191.

Challamel, N. (2000). Rock destruction effect on the stability of a drilling structure, Journal of Sound and Vibration 233(2): 235-254.

Detournay, E. and Defourny, P. (1992). A phenomenological model for the drilling action of drag bits, International 
Journal of Rock Mechanics, Mining Science and Geomechanical Abstracts 29(1): 13-23.

Fliess, M., Lévine, J., Martin, P. and Rouchon, P. (1995). Flatness and defect of non-linear systems: Introductory theory and examples, International Journal of Control 61(6): 1327-1361.

Fridman, E. and Dambrine, M. (2010). Control under quantization, saturation and delay: A LMI approach, $\mathrm{Au}$ tomatica 45(10): 2258-2264.

Fridman, E., Dambrine, M. and Yeganefar, N. (2008). Input to state stability of systems with time-delay: A matrix inequalities approach, Automatica 44(9): 2364-2369.

Fridman, E., Mondié, S. and Saldivar, B. (2010). Bounds on the response of a drilling pipe model, IMA Journal of Mathematical Control and Information 27(4): 513-526.

Grujić, L.T. (1973). On practical stability, International Journal of Control 17(4): 881-887.

Halsey, G., Kyllingstad, A. and Kylling, A. (1988). Torque feedback used to cure slip-stick motion, Proceedings of the 63rd Society of Petroleum Engineers Drilling Engineering Annual Technical Conference and Exhibition, Houston, TX, USA, pp. 277-282.

Jansen, J. (1993). Nonlinear Dynamics of Oilwell Drillstrings, Ph.D. thesis, Delft University of Technology, Delft.

Jansen, J. and van den Steen, L. (1995). Active damping of self-excited torsional vibrations in oil well drillstrings, Journal of Sound and Vibration 179(4): 647-668.

Javanmardi, K. and Gaspard, D. (1992). Application of soft torque rotary table in mobile bay, Technical Report IADC/SPE 23913, International Association of Drilling Contractors/Society of Petroleum Engineers, Dallas, TX.

Khalil, H. (2002). Nonlinear Systems, Third Edition, Prentice-Hall, Upper Saddle River, NJ.

Knuppel, T., Woittennek, F., Boussaada, I., Mounier, H. and Niculescu, S. (2014). Flatness-based control for a non-linear spatially distributed model of a drilling system, in A. Seuret et al. (Eds.), Low Complexity Controllers for Time Delay Systems: Advances in Delays and Dynamics, Volume 2, Springer, Cham, pp. 205-218.

Kǒcvara, M. and Stingl, M. (2003). PENNON-a code for nonlinear and convex semidefinite programming, Optimization Methods and Software 8(3): 317-333.

La Salle, J. and Lefschetz, S. (1961). Stability by Lyapunov's Direct Method: With Applications, Academic Press, New York, NY.

Lakshmikantham, V., Leela, S. and Martynyuk, A. (1990). Practical Stability of Nonlinear Systems, World Scientific Publishing Company, Singapore.

Levinson, N. (1944). Transformation theory of non-linear differential equations of the second order, Annals of Mathematics 45(4): 723-737.

Lu, H., Dumon, J. and de Wit, C.C. (2009). Experimental study of the D-OSKIL mechanism for controlling the stick-slip oscillations in a drilling laboratory testbed, 2009 IEEE Control Applications (CCA) \& Intelligent Control (ISIC), St. Petersburg, Russia, pp. 1551-1556.
Ma, R., Dimirovski, G. and Zhao, J. (2013). Backstepping robust $\mathcal{H}_{\infty}$ control for a class of uncertain switched nonlinear systems under arbitrary switchings, Asian Journal of Control 15(1): 41-50.

Navarro-López, E. and Cortés, D. (2007a). Avoiding harmful oscillations in a drillstring through dynamical analysis, Journal of Sound and Vibration 307(1): 152-171.

Navarro-López, E. and Cortés, D. (2007b). Sliding-mode control of a multi-DOF oilwell drillstring with stick-slip oscillations, Proceedings of the 2007 American Control Conference, New York, NY, USA, pp. 3837-3842.

Navarro-López, E. and Licéaga-Castro, E. (2009). Non-desired transitions and sliding-mode control of a multi-DOF mechanical system with stick-slip oscillations, Chaos, Solitons and Fractals 41(4): 2035-2044.

Navarro-López, E. and Suárez, R. (2004). Practical approach to modelling and controlling stick-slip oscillations in oilwell drillstrings, Proceedings of the 2004 IEEE International Conference on Control Applications Taipei, Taiwan, pp. 1454-1460.

Pavone, D. and Desplans, J. (1994). Application of high sampling rate downhole measurements for analysis and cure of stick-slip in drilling, Technical Report SPE 28324, Society of Petroleum Engineers, Dallas, TX.

Rasvan, V. (2006). Three lectures on dissipativeness, IEEE International Conference on Automation, Quality and Testing, Robotics, Cluj-Napoca, Romania, pp. 167-177.

Saldivar, B., Knuppel, T., Woittennek, F., Boussaada, I., Mounier, H. and Niculescu, S. (2014). Flatness-based control of torsional-axial coupled drilling vibrations, 19th World Congress of the International Federation of Automatic Control, Cape Town, South Africa, pp. 7324-7329.

Saldivar, B. and Mondié, S. (2013). Drilling vibration reduction via attractive ellipsoid method, Journal of the Franklin Institute 350(3): 485-502.

Saldivar, B., Mondié, S., Loiseau, J. and Rasvan, V. (2013). Suppressing axial torsional coupled vibrations in oilwell drillstrings, Journal of Control Engineering and Applied Informatics 15(1): 3-10.

Serrarens, A., van de Molengraft, M., Kok, J. and van den Steeen, L. (1998). $\mathcal{H}_{\infty}$ control for suppressing stick-slip in oil well drillstrings, IEEE Control Systems 18(2): 19-30.

Skaugen, E. (1987). The effects of quasi-random drill bit vibrations upon drillstring dynamic behavior, Technical Report SPE 16660, Society of Petroleum Engineers, Dallas, TX.

Suh, Y., Kang, H. and Ro, Y. (2006). Stability condition of distributed delay systems based on an analytic solution to Lyapunov functional equations, Asian Journal of Control 8(1): 91-96.

Timoshenko, S. and Young, D. (1955). Vibrations Problems in Engineering, Third Edition, D. Van Nostrand Company, Princeton, NJ.

Tucker, R. and Wang, C. (1999). On the effective control of torsional vibrations in drilling systems, Journal of Sound and Vibration 224(1): 101-122. 
Weaver, W., Timoshenko, S. and Young, D. (1990). Vibrations Problems in Engineering, Fifth Edition, John Wiley \& Sons, New York, NY.

Wu, J., Li, S. and Chai, S. (2010). Exact controllability of wave equations with variable coefficients coupled in parallel, Asian Journal of Control 12(5): 650-655.

Yang, L. and Wang, J. (2014). Stability of a damped hyperbolic Timoshenko system coupled with a heat equation, Asian Journal of Control 16(2): 546-555.

Yoshizawa, T. (1960). Stability and boundedness of systems, Archive for Rational Mechanics and Analysis 6(1): 409-421.

Yoshizawa, T. (1966). Stability Theory by Lyapunov's Second Method, The Mathematical Society of Japan, Tokyo.

Zhang, X. and Zuazua, E. (2004). Polynomial decay and control of a 1-D hyperbolic-parabolic coupled system, Journal of Differential Equations 204(2): 380-438.

Zhou, Z. and Tang, S. (2012). Boundary stabilization of a coupled wave-ode system with internal anti-damping, International Journal of Control 85(11): 683-693.

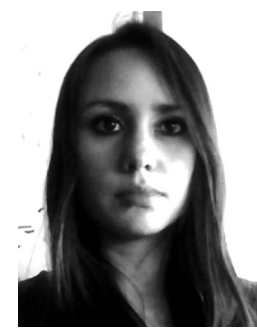

Belem Saldivar received a B.Sc. degree in electronics and telecommunications engineering from UAEH, Pachuca Hidalgo, Mexico, in 2007. She obtained an M.Sc. degree in automatic control from CINVESTAV, Mexico City, Mexico, in 2010 and Ph.D. degrees in automatic control as well as informatics and applications from CINVESTAV and IRCCyN, Nantes, France, in 2013. Since 2014 she has been a CONACYT research fellow at the Autonomous University of the State of Mexico (UAEM). Her research is focused on the modeling, analysis, and control of dynamical systems.

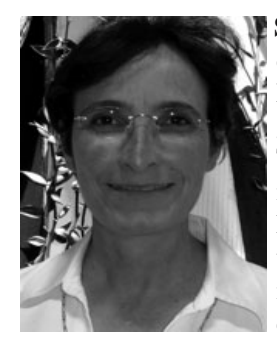

Sabine Mondié received the B.Sc. degree in industrial engineering from ITESM, Mexico City, Mexico, in 1981, and the M.S. and Ph.D. degrees in electrical engineering from CINVESTAV, Mexico City, and IRCCyN, Nantes, France, in 1983 and 1996, respectively. Since 1996, she has been a professor at the Department of $\mathrm{Au}$ tomatic Control of CINVESTAV. Her research is focused on time delay systems, their analysis, control and applications.

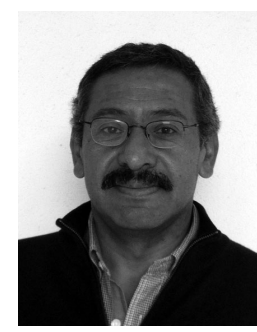

Juan Carlos Ávila Vilchis is a mechanical engineering graduate of the Autonomous University of the State of Mexico (UAEM). He received a mechanical engineering master's degree from the Industrial Institute of the Nord (IDN, now Lille Central School, France) in 1990 and the doctoral degree from the Polytechnic National Institute of Grenoble, France, in 2001. Since 2003, he has been working at the UAEM postgraduate department. His research interests are nonlinear systems and medical applications of robotics.

\section{Appendix}

\section{Proof of Lemma 1}

Multiplying (10) by e ${ }^{\sigma \theta}$ gives

$$
\frac{\mathrm{d}}{\mathrm{d} \theta}\left[\mathrm{e}^{\sigma \theta} V(\tilde{z}(\cdot, \theta))\right] \leq \beta \varepsilon \mathrm{e}^{\sigma \theta},
$$

and integration of the above expression from $t_{0}$ to $t$ yields

$$
\mathrm{e}^{\sigma t} V(\tilde{z}(\cdot, t))-\mathrm{e}^{\sigma t_{0}} V\left(\tilde{\phi}\left(\zeta, t_{0}\right)\right) \leq \frac{\beta \varepsilon}{\sigma}\left[\mathrm{e}^{\sigma t}-\mathrm{e}^{\sigma t_{0}}\right] .
$$

Without loss of generality, we assume $t_{0}=0$; then we obtain

$$
V(\tilde{z}(\cdot, t)) \leq \mathrm{e}^{-\sigma t} V\left(\tilde{\phi}\left(\zeta, t_{0}\right)\right)+\frac{\beta \varepsilon}{\sigma}\left[1-\mathrm{e}^{-\sigma t}\right] .
$$

It follows from (9) that

$$
\begin{aligned}
\int_{0}^{1} \tilde{z}^{T}(\zeta, t) P \tilde{z}(\zeta, t) \mathrm{d} \zeta \leq & \mathrm{e}^{-\sigma t} \bar{V}\left(\tilde{\phi}\left(\zeta, t_{0}\right)\right) \\
& +\frac{\beta \varepsilon}{\sigma}\left[1-\mathrm{e}^{-\sigma t}\right]
\end{aligned}
$$

Then we have

$$
\begin{aligned}
\int_{0}^{1} \tilde{z}^{T}(\zeta, t) P \tilde{z}(\zeta, t) \mathrm{d} \zeta \leq & \mathrm{e}^{-\sigma t}\left[\bar{V}\left(\tilde{\phi}\left(\zeta, t_{0}\right)\right)-\frac{\beta \varepsilon}{\sigma}\right] \\
& +\frac{\beta \varepsilon}{\sigma} .
\end{aligned}
$$

For the initial conditions fulfilling $\bar{V}\left(\tilde{\phi}\left(\zeta, t_{0}\right)\right) \leq \beta \varepsilon / \sigma$,

$$
\int_{0}^{1} \tilde{z}^{T}(\zeta, t) P \tilde{z}(\zeta, t) \mathrm{d} \zeta \leq \frac{\beta \varepsilon}{\sigma}, \quad \forall t \geq 0
$$

otherwise, if $\bar{V}\left(\tilde{\phi}\left(\zeta, t_{0}\right)\right)>\beta \varepsilon / \sigma$, we look for a time instant $T_{a}(\tilde{\phi}, \varsigma)$ at which the ultimate bound (11) is satisfied.

In view of (A1), $T_{a}(\tilde{\phi}, \varsigma)$ should guarantee $\int_{0}^{1} \tilde{z}^{T}(\zeta, t) P \tilde{z}(\zeta, t) \mathrm{d} \zeta \leq \varsigma+\beta \varepsilon / \sigma$ for $\varsigma>0$, small enough, i.e.,

$$
\varsigma=\mathrm{e}^{-\sigma T_{a}}\left[\bar{V}(\tilde{\phi}(\zeta, t))-\frac{\beta \varepsilon}{\sigma}\right] .
$$

From the above expression we have

$$
\mathrm{e}^{\sigma T_{a}}=\frac{1}{\varsigma}\left[\bar{V}(\tilde{\phi}(\zeta, t))-\frac{\beta \varepsilon}{\sigma}\right],
$$

and the result follows. 